\title{
Adaptive Control of a New Chaotic Financial System with Integer Order and Fractional Order and Its Identical Adaptive Synchronization
}

\author{
Paul Yaovi Dousseh, ${ }^{1}$ Cyrille Ainamon, ${ }^{1}$ Clément Hodévèwan Miwadinou $\mathbb{D}^{1,2}$ \\ Adjimon Vincent Monwanou, ${ }^{1}$ and Jean Bio Chabi Orou ${ }^{1}$ \\ ${ }^{1}$ Laboratoire de Mécaniques des Fluides, de la Dynamique Non-linéaire et de la Modélisation des Systèmes \\ Biologiques (LMFDNMSB), Institut de Mathématiques et de Sciences Physiques (IMSP), Porto-Novo, Benin \\ ${ }^{2}$ Département de Physique, ENS-Natitingou, Université des Sciences, Technologies, Ingénierie et Mathématiques (UNSTIM), \\ Abomey, Benin \\ Correspondence should be addressed to Clément Hodévèwan Miwadinou; clement.miwadinou@imsp-uac.org
}

Received 19 January 2021; Revised 13 February 2021; Accepted 26 February 2021; Published 13 March 2021

Academic Editor: Yi Qi

Copyright (c) 2021 Paul Yaovi Dousseh et al. This is an open access article distributed under the Creative Commons Attribution License, which permits unrestricted use, distribution, and reproduction in any medium, provided the original work is properly cited.

\begin{abstract}
In this paper, adaptive control and adaptive synchronization of an integer and fractional order new financial system with unknown constant parameters are studied. Based on Lyapunov's stability theory, an adaptive control law is designed to asymptotically stabilize the state variables of the system to the origin in integer and fractional order cases. By the same theory, an adaptive synchronization law is designed to perform the identical synchronization of the new financial system in the cases of integer and fractional order with unknown constant parameters. Numerical simulations are carried out in order to show the efficiency of the theoretical results.
\end{abstract}

\section{Introduction}

Fractional order derivatives are a subject over 300 years old, initiated by Leibniz's letter to L'Hospital $[1,2]$ and are a generalization of integer order derivatives But their applications in scientific fields are very recent and this is due to the lack of their physical interpretation. The difference between these fractional order derivatives and the integer order derivatives is that fractional order derivatives have the memory that turns out to be very useful when it comes to describing systems with memory and heredity properties. In the literature, several systems have been described using fractional order derivatives, we can cite the fractional order Liu system [3], the fractional order financial system [4], the fractional order glucose-insulin regulatory system [5], the fractional order Chua system [6], etc. Chaotic dynamical systems are first of all nonlinear systems, depending on several parameters and having an extreme sensitivity to initial conditions. These systems are found in many scientific fields including chemical, physical [6], economic [4], or biological [5]. This has led researchers from various horizons to take an interest in these types of systems, and especially the control of the chaos which intervenes and the synchronization of these systems with integer and fractional order. Chaos control in a dynamical system consists in designing a control law which stabilizes the system asymptotically on one of these unstable fixed points. In the literature, several methods have been proposed to achieve this goal. We have among others, the linear feedback control [7], adaptive control [8,9], sliding mode control [10], Lyapunov-based nonlinear control [11], adaptive sliding mode control [12], etc. Recently, for the stabilization of dynamical systems, different results have been obtained in the literature in fields as diverse as varied. For example, see 
[13], where authors proceeded to the stabilization of a class of chaotic systems when systems are subject to uncertainty and external disturbance by a new uncertainty and disturbance estimator- (UDE-) based control method. In [14], a novel distributed consensus algorithm based on the integration of sliding mode control scheme and (average) ADT method is proposed to solve consensus control problem in order to guarantee the stability of the closed-loop system. Also, in [15], the finite horizon control for a broad class of linear It ôstochastic differential equations (SDEs) with infinite Markovian jumps and $(x, u, v)$-dependent noise is done. The authors proposed the existence of the mixed control, a necessary and sufficient condition, which is represented by the solution of a countably infinite set of coupled generalized difference Riccati equations (GDREs).

The synchronization of integer and fractional order systems has also been widely discussed in the literature due to its applications in the field of communication $[17,18]$ for the secure transmission of information. Several approaches are used for the synchronization of chaotic systems such as synchronization via nonlinear control $[19,20]$, synchronization via active control $[7,21,22]$, and adaptive synchronization $[8,9,23]$. The particularity of adaptive control and adaptive synchronization is that these unlike other controllers, which are used when the system's parameters are known, are used when the system's parameters are unknown.

In the field of economics, several models have been proposed [24, 25]. The study of dynamic behavior, and the control of chaos in financial and economic systems have also been approached in order to understand the dynamic behavior of these systems and stabilize them in order to eliminate undesirable behavior [10, 26-31]. In 2020, Liao et al. [32] presented a new model to take into account the interaction between the various state variables of the system. The numerical study of this model revealed that it presents complex dynamic behaviors such as period doubling and chaos [32]. It would therefore be interesting to control the chaos in this new financial system, in other words, to eliminate the undesirable behaviors of the system by considering the case where constant parameters of the system are unknown and also to carry out the identical adaptive synchronization of this new chaotic system. It is in this context that this work is part of which in order to control the chaotic behavior of the new financial system when the parameters are unknown; an adaptive control law will be designed to stabilize asymptotically at the origin the state variables of the integer and fractional order system. The case of the adaptive synchronization of the new financial system with integer and fractional order will also be discussed.

The organization of the rest of this paper is as follows: in Section 2, some concepts on fractional calculus and the description of the new financial system with integer and fractional order are given. The adaptive control of chaos in the new financial system based on Lyapunov's stability theory in the cases of integer order and incommensurate fractional order are done in Section 3. Section 4 deals with the adaptive synchronization of the new financial system in the cases of integer and incommensurate fractional order. Finally, the conclusion is discussed in Section 5.

\section{Some Fractional Calculus Concepts and Model Description}

The arbitrary order derivative, in other words, the fractional order derivative, is a generalization of the integer order derivative or the classical derivative. We generally meet in the literature three definitions of fractional order derivative [33]. In this paper, we will use the fractional order derivative in the sense of Caputo because with this derivative, the initial conditions take the same form as when the system is defined with integer order derivative.

The fractional order derivative in the sense of Caputo $(C)$ is defined by

$$
{ }_{a}^{C} D_{t}^{q} f(t)=\frac{1}{\Gamma(n-1)} \int_{a}^{t}(t-\tau)^{n-q-1} f^{(n)}(\tau) \mathrm{d} \tau, \quad n-1<q<n,
$$

where $\Gamma(\cdot)$ is the gamma function and $q$ is the order of the fractional derivative.

The fractional order derivative in the sense of Caputo has a certain number of properties defined as follows $[33,34]$.

Property 1. Suppose that $0<q<1$, then

$$
D y(t)=D^{1-q} D^{q} y(t)
$$

in which $D=(\mathrm{d} / \mathrm{d} t)$.

Property 2. When $q=0$,

$$
D^{0} y(t)=y(t)
$$

Property 3. As in the case of the integer order derivative, the fractional order derivative in the sense of Caputo is a linear operator:

$$
D^{q}(\gamma x(t)+\delta y(t))=\gamma D^{q} x(t)+\delta D^{q} y(t),
$$

in which $\gamma$ and $\delta$ are real constants.

Property 4. As in the case of the integer order derivative, the fractional order derivative in the sense of Caputo satisfies the additive index law, i. e.,

$$
D^{q_{1}} D^{q_{2}} y(t)=D^{q_{2}} D^{q_{1}} y(t)=D^{q_{1}+q_{2}} y(t)
$$

with some reasonable constraints on the function $y(t)$.

In 2020, Liao et al. [32] presented a new financial model in order to take into account the interaction between the interest rate $x(t)$, the investment demand $y(t)$, and the price index $z(t)$. The system is defined as follows: 


$$
\left\{\begin{array}{l}
\frac{\mathrm{d} x}{\mathrm{~d} t}=\mathrm{d} z+(y-e) x \\
\frac{\mathrm{d} y}{\mathrm{~d} t}=-k y^{2}-l x^{2}+m \\
\frac{\mathrm{d} z}{\mathrm{~d} t}=-\gamma z-\delta x-\rho y
\end{array}\right.
$$

where the parameters $e, k, \gamma, m, l, \rho$, and $\delta$ are constants. In [32], when $e=0.3, k=0.02, \gamma=1, m=1, l=0.1, \rho=$ 0.05 , and $d=1.2, \delta=1$ and initial conditions $(1.2,1.5,1.6)$ are considered, system (6) exhibits a chaotic behavior as shown in Figures 1(a)-1(d). When the initial conditions (0.2, 0.5 , and 0.6 ) are considered, system (6) also presents a chaotic behavior [32].

The generalization of system (6), i.e., the fractional order version of the new financial system is also considered in this study. Classical derivatives (integer order) are replaced by fractional order derivatives as follows:

$$
\left\{\begin{array}{l}
D^{q_{1}} x=d z+(y-e) x \\
D^{q_{2}} y=-k y^{2}-l x^{2}+m \\
D^{q_{3}} z=-\gamma z-\delta x-\rho y
\end{array}\right.
$$

where $q_{i} \in(0,1)$ and $D^{q_{i}}=\left(\mathrm{d}^{q_{i}} / \mathrm{d} t^{q_{i}}\right)(i=1,2,3)$. If $q_{1}=q_{2}=q_{3}=q$, then system (7) is said to be a commensurate order system; otherwise, it is said to be an incommensurate order system.

The new fractional order financial system is chaotic when the values of the above parameters are considered; the initial conditions $(1.2,1.5,1.6)$ and the orders $q_{1}=1, q_{2}=0.88$, and $q_{3}=1$ are considered.

The phase diagrams projected onto the phase planes $(x, y),(x, z)$, and the time histories of the state variables $x(t)$ and $y(t)$ are shown in Figures 2(a)-2(d).

\section{Adaptive Control of Chaos in a New Financial System}

3.1. Integer Order Case. In this part, we will be interested in the design of an adaptive control law in order to globally stabilize the new integer order financial system.

3.1.1. Controller Design. To control the chaos in system (6), adaptive controllers are added to it. The new controlled financial system can therefore be written in the following form:

$$
\left\{\begin{array}{l}
\dot{x}=d z+(y-e) x+u_{1}, \\
\dot{y}=-k y^{2}-l x^{2}+m+u_{2}, \\
\dot{z}=-\gamma z-\delta x-\rho y+u_{3},
\end{array}\right.
$$

in which $\dot{x}=(\mathrm{d} x / \mathrm{d} t), \dot{y}=(\mathrm{d} y / \mathrm{d} t)$, and $\dot{z}=(\mathrm{d} z / \mathrm{d} t)$. The $u_{i},(i=1,2,3)$ are adaptive controllers which will be subsequently designed taking into account the state variables of the system and the estimation of the unknown constant parameters $d, e, k, l, m, \gamma, \delta$, and $\rho$ of the system.
To allow the state variables of the system to converge asymptotically to the origin, we take the following adaptive control functions:

$$
\left\{\begin{array}{l}
u_{1}=-y x-\widehat{d} z+\widehat{e} x-h_{1} x \\
u_{2}=\widehat{k} y^{2}+\widehat{l} x^{2}-\widehat{m}-h_{2} y \\
u_{3}=\widehat{\gamma} z+\widehat{\delta} x+\widehat{\rho} y-h_{3} z
\end{array}\right.
$$

in which $\hat{d}, \widehat{e}, \widehat{k}, \hat{l}, \widehat{m}, \widehat{\gamma}, \widehat{\delta}$, and $\hat{\rho}$ are the estimation of the unknown constant parameters $d, e, k, l, m, \gamma, \delta$, and $\rho$, respectively, and $h_{i},(i=1,2,3)$ are positive constants.

By replacing the control law (9) in system (8), we have

$$
\left\{\begin{array}{l}
\dot{x}=(d-\widehat{d}) z-(e-\widehat{e}) x-h_{1} x \\
\dot{y}=-(k-\widehat{k}) y^{2}-(l-\widehat{l}) x^{2}+(m-\widehat{m})-h_{2} y \\
\dot{z}=-(\gamma-\widehat{\gamma}) z-(\delta-\widehat{\delta}) x-(\rho-\widehat{\rho}) y-h_{3} z .
\end{array}\right.
$$

Let us define the estimation error of unknown parameters as follows:

$$
\left\{\begin{array}{l}
e_{d}=d-\widehat{d}, \\
e_{e}=e-\widehat{e}, \\
e_{k}=k-\widehat{k}, \\
e_{l}=l-\widehat{l}, \\
e_{m}=m-\widehat{m}, \\
e_{\gamma}=\gamma-\widehat{\gamma}, \\
e_{\delta}=\delta-\widehat{\delta}, \\
e_{\rho}=\rho-\widehat{\rho} .
\end{array}\right.
$$

By replacing equation (11) in system (10), we have

$$
\left\{\begin{array}{l}
\dot{x}=e_{d} z-e_{e} x-h_{1} x \\
\dot{y}=-e_{k} y^{2}-e_{l} x^{2}+e_{m}-h_{2} y \\
\dot{z}=-e_{\gamma} z-e_{\delta} x-e_{\rho} y-h_{3} z
\end{array}\right.
$$

For the design of the parameter update law which will allow to adjusting the parameter estimates, we use Lyapunov's stability theory.

For this, consider the Lyapunov quadratic function defined as follows:

$$
V=\frac{1}{2}\left(x^{2}+y^{2}+z^{2}+e_{d}^{2}+e_{e}^{2}+e_{k}^{2}+e_{l}^{2}+e_{m}^{2}+e_{\gamma}^{2}+e_{\delta}^{2}+e_{\rho}^{2}\right)
$$

which is a positive definite function on $R^{11}$.

The derivative with respect to time of equation (13) gives us

$$
\begin{aligned}
\dot{V}= & x \dot{x}+y \dot{y}+z \dot{z}+e_{d} \dot{e}_{d}+e_{e} \dot{e}_{e}+e_{k} \dot{e}_{k} \\
& +e_{l} \dot{e}_{l}+e_{m} \dot{e}_{m}+e_{\gamma} \dot{e}_{\gamma}+e_{\delta} \dot{e}_{\delta}+e_{\rho} \dot{e}_{\rho}
\end{aligned}
$$

which specify that 


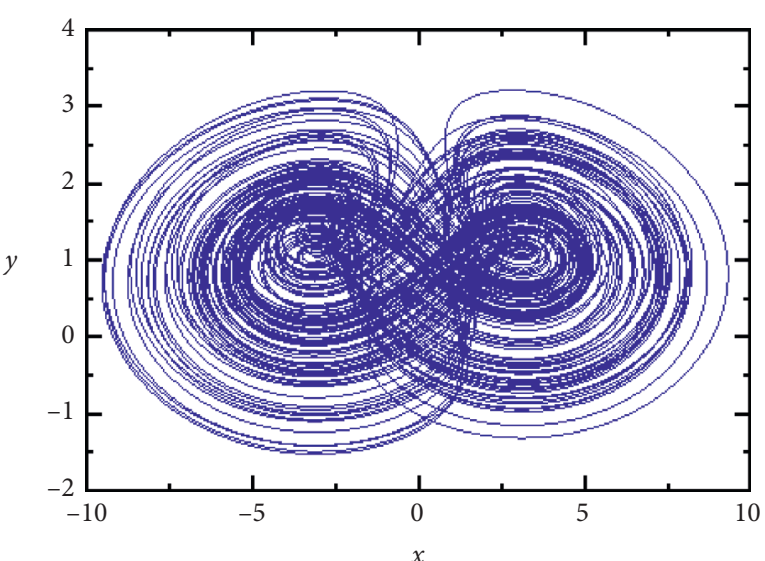

(a)

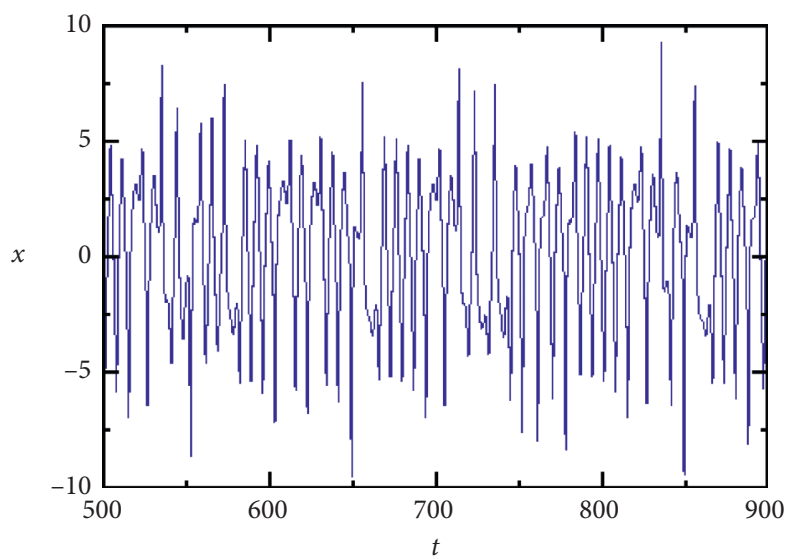

(c)

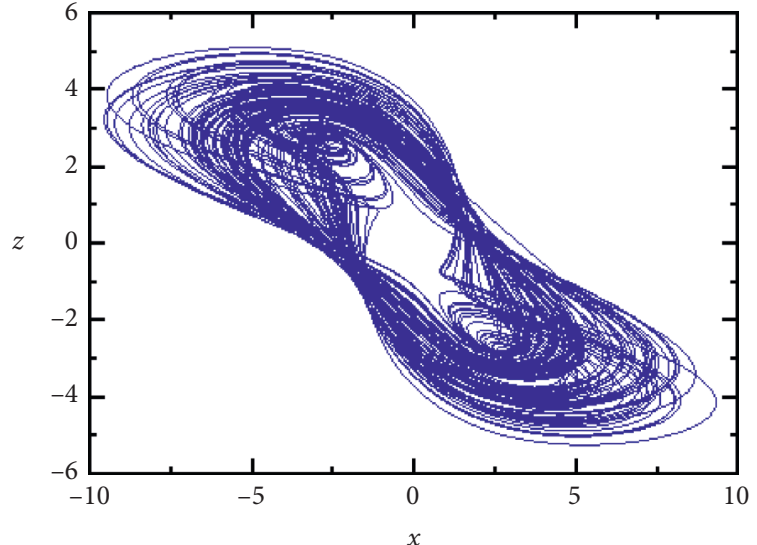

(b)

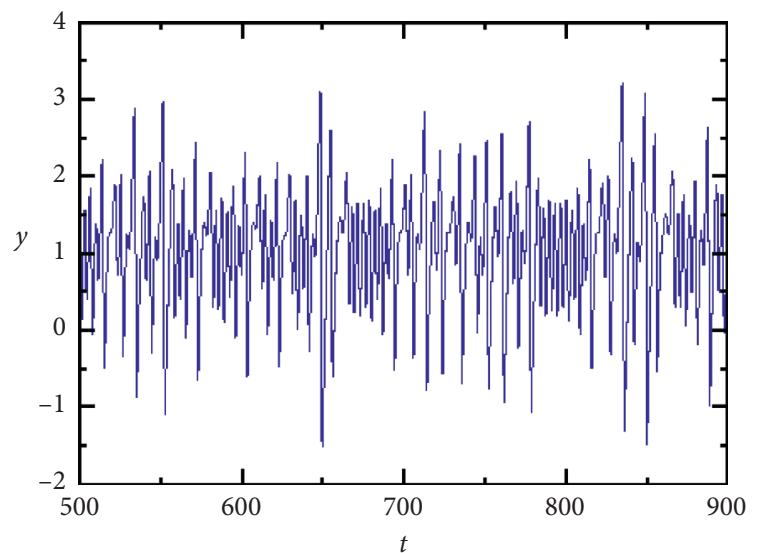

(d)

Figure 1: Phase diagrams and time histories of system (6): (a) projected onto $x$-y phase plane, (b) projected onto $x$ - $z$ phase plane, (c) time history of $(x)$, and (d) time history of $y$.

$$
\left\{\begin{array}{l}
\dot{e}_{d}=-\dot{\hat{d}} \\
\dot{e}_{e}=-\dot{\hat{e}} \\
\dot{e}_{k}=-\dot{\hat{k}} \dot{e}_{l}=-\dot{\hat{l}}, \dot{e}_{m}=-\dot{\hat{m}}, \dot{e}_{\gamma}=-\dot{\hat{\gamma}}, \dot{e}_{\delta}=-\dot{\widehat{\delta}}, \dot{e}_{\rho}=-\dot{\hat{\rho}} .
\end{array}\right.
$$

Using system (12) and equation (15), equations (14) becomes

$$
\begin{aligned}
\dot{V}= & -h_{1} x^{2}-h_{2} y^{2}-h_{3} z^{2}+e_{d}(x z-\dot{\hat{d}}) \\
& +e_{e}\left(-x^{2}-\dot{\hat{\hat{e}}}\right)+e_{k}\left(-y^{3}-\dot{\hat{\hat{k}}}\right) \\
& +e_{l}\left(-y x^{2}-\dot{\hat{l}}\right)+e_{m}(y-\dot{\hat{\mathrm{m}}}) \\
& +e_{\gamma}\left(-z^{2}-\dot{\hat{\gamma}}\right)+e_{\delta}(-x z-\dot{\hat{\delta}}) \\
& +e_{\rho}(-z y-\dot{\hat{\rho}})
\end{aligned}
$$

From equation (16), we deduce that the estimated parameters update law is

$$
\left\{\begin{array}{l}
\dot{\hat{d}}=x z+h_{4} e_{d}, \\
\dot{\hat{e}}=-x^{2}+h_{5} e_{e}, \\
\dot{\hat{k}}=-y^{3}+h_{6} e_{k}, \\
\dot{\hat{l}}=-y x^{2}+h_{7} e_{l}, \\
\dot{\hat{m}}=y+h_{8} e_{m}, \\
\dot{\hat{\gamma}}=-z^{2}+h_{9} e_{\gamma}, \\
\dot{\hat{\delta}}=-x z+h_{10} e_{\delta}, \dot{\hat{\rho}}=-z y+h_{11} e_{\rho},
\end{array}\right.
$$

in which $h_{4}, h_{5}, h_{6}, h_{7}, h_{8}, h_{9}, h_{10}$, and $h_{11}$ are positive constants.

By replacing equation (17) in (16), we have

$$
\begin{aligned}
\dot{V}= & -h_{1} x^{2}-h_{2} y^{2}-h_{3} z^{2}-h_{4} e_{d}^{2} \\
& -h_{5} e_{e}^{2}-h_{6} e_{k}^{2}-h_{7} e_{l}^{2}-h_{8} e_{m}^{2}-h_{9} e_{\gamma}^{2} \\
& -h_{10} e_{\delta}^{2}-h_{11} e_{\rho}^{2}<0,
\end{aligned}
$$

which is the negative definite function on $R^{11}$ for positive constants $h_{i},(i=1, \ldots, 11)$. 


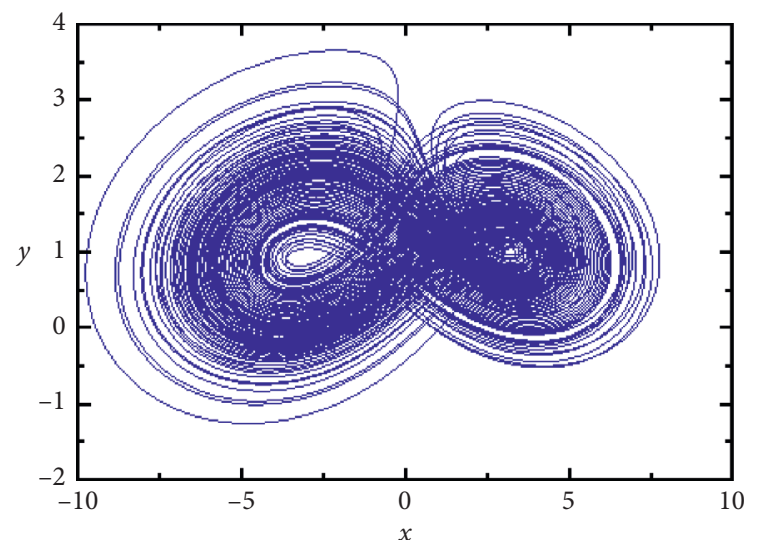

(a)

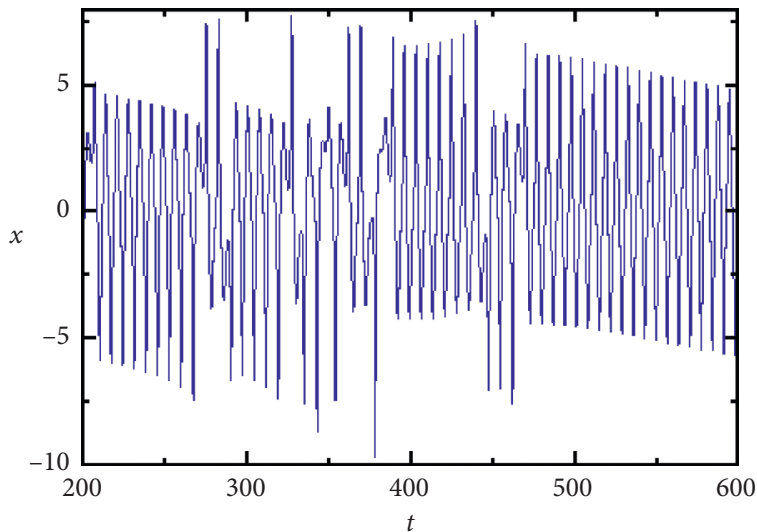

(c)

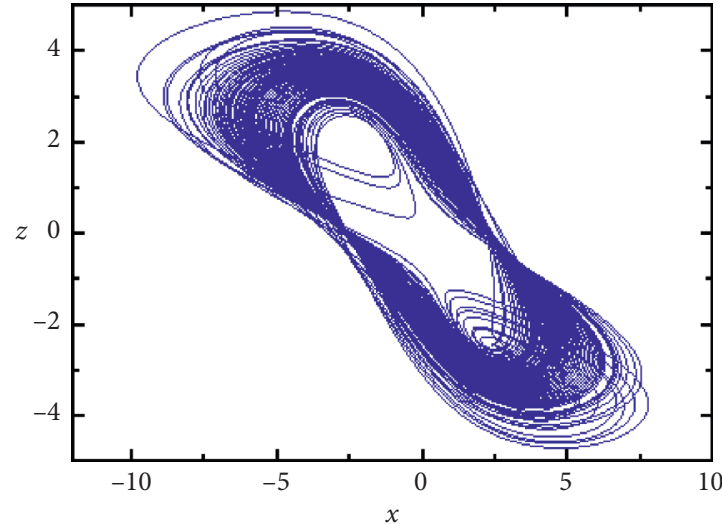

(b)

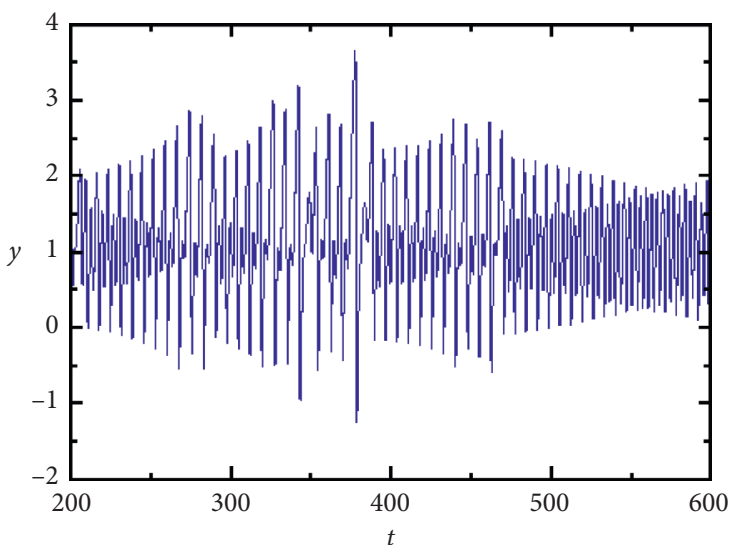

(d)

Figure 2: Phase diagrams and time histories of system (7) with $q_{1}=1, q_{2}=0.88$, and $q_{3}=1$ : (a) projected onto $x$-y phase plane, (b) projected onto $x-z$ phase plane, (c) time history of $(x)$, and (d) time history of $y$.

So, we have found a function which verifies the Lyapunov stability theorem $(V>0, \dot{V}<0)$.

Thus, we have the following result.

Theorem 1. The new financial system (8) with the unknown parameters is globally and asymptotically stabilized at the origin for all initial conditions by the adaptive law (9), where the update law of the parameters is given by (17) with $h_{i},(i=$ $1, \ldots, 11)$ being positive constants.

3.1.2. Simulation Results. In this part, we use the fourthorder Runge-Kutta algorithm to solve the new financial system (8) with the adaptive law (9) and the parameters update law (17). For the simulation, the time-step $h=0.001$ is chosen. The initial conditions $(x(0), y(0), z(0))=$ $(1.2,1.5,1.6)$ are used. The parameters of the new financial system are chosen as follows:

$e=0.3, k=0.02, \gamma=1, m=1, l=0.1, \rho=0.05, d=1.2, \delta=1$.

For $h_{i},(1, \ldots, 11)$ of the adaptive and update laws, we choose $h_{i}=3$.

For the initial value of the estimated parameters, we assume the following values:

$$
\hat{e}(0)=3, \hat{k}(0)=1, \hat{\gamma}(0)=4, \hat{m}(0)=3, \hat{l}(0)=1, \hat{\rho}(0)=2, \hat{d}(0)=1, \hat{\delta}(0)=3 \text {. }
$$

By applying the adaptive control law (9) and the parameter update law (17) to the new controlled financial system (8), the results of the numerical simulations are shown in
Figures 3 and 4. From Figure 3, it can be seen that the state variables of the system converge asymptotically towards the origin (zero). Figure 4 shows the estimated parameters for 

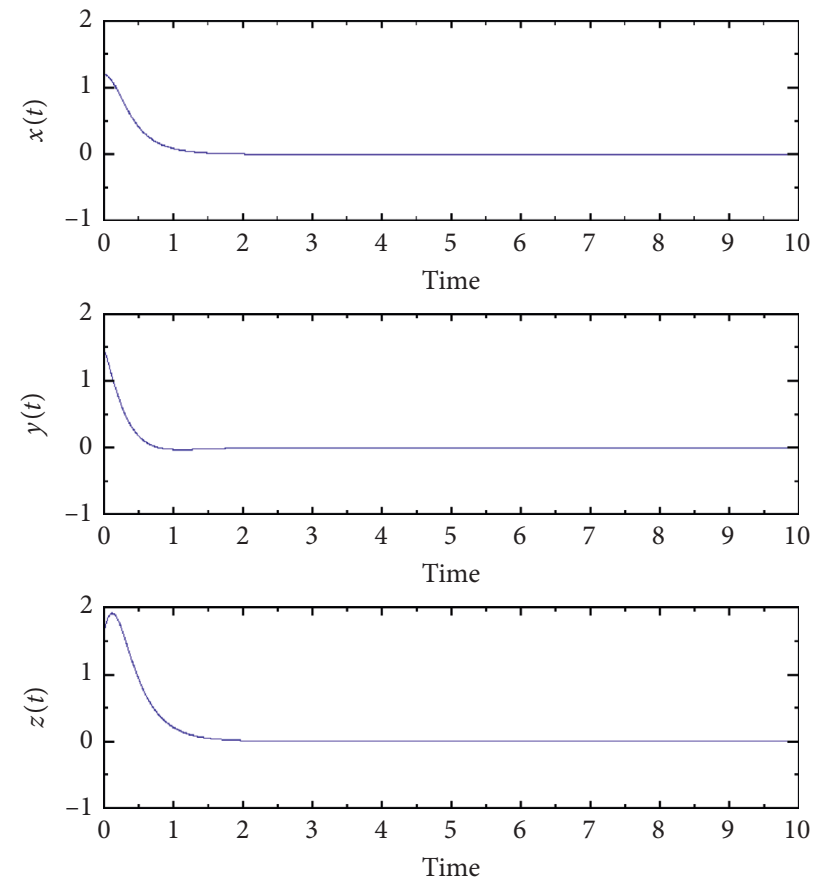

Figure 3: Time histories of the controlled integer order new financial system (8).

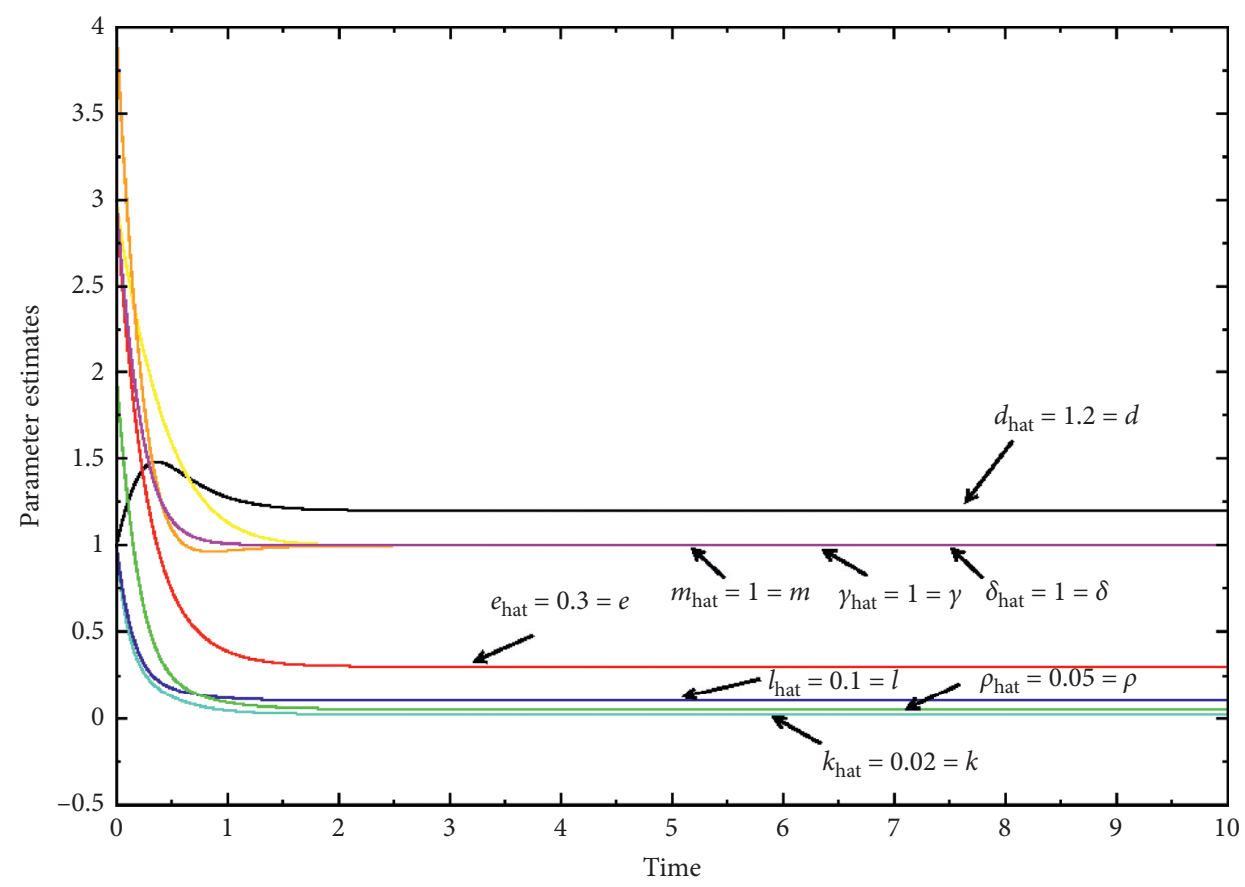

FIGURE 4: Parameter estimates for adaptive control in integer order case. 
adaptive control in integer order case and, as it can be seen, these parameters converge towards the real values of the parameters of the system, i.e.,

$e=0.3, k=0.02, \gamma=1, m=1, l=0.1, \rho=0.05, d=1.2, \delta=1$.

3.2. Fractional Order Case. In this part, the adaptive control of the new fractional order financial system is performed to globally stabilize the new financial system with fractional order.

3.2.1. Controller Design. Consider the following new fractional order controlled financial system:

$$
\left\{\begin{array}{l}
D^{q_{1}} x=d z+(y-e) x+u_{1} \\
D^{q_{2}} y=-k y^{2}-l x^{2}+m+u_{2} \\
D^{q_{3}} z=-\gamma z-\delta x-\rho y+u_{3}
\end{array}\right.
$$

in which the $u_{i},(i=1,2,3)$ are adaptive controllers which will be subsequently designed taking into account the sate variables of the system and the estimation of the unknown constant parameters $d, e, k, l, m, \gamma, \delta$, and $\rho$ of the system.

To allow the states of the system to converge asymptotically to the origin, we take the following adaptive control functions:

$$
\left\{\begin{array}{l}
u_{1}=-y x-\hat{d} z+\widehat{e} x-h_{1} D^{q_{1}-1} x+v_{1}, \\
u_{2}=\widehat{k} y^{2}+\widehat{l} x^{2}-\widehat{m}-h_{2} D^{q_{2}-1} y+v_{2}, \\
u_{3}=\widehat{\gamma} z+\widehat{\delta} x+\widehat{\rho} y-h_{3} D^{q_{3}-1} z+v_{3}
\end{array}\right.
$$

in which $\widehat{d}, \widehat{e}, \widehat{k}, \widehat{l}, \widehat{m}, \widehat{\gamma}, \widehat{\delta}$, and $\widehat{\rho}$ are the estimation of the unknown constant parameters $d, e, k, l, m, \gamma, \delta$, and $\rho$, respectively. The $h_{i},(i=1,2,3)$ are positive constants and $v_{i},(i=1,2,3)$ are nonlinear functions that will be designed.

By replacing the control law (23) in system (22), we have

$$
\left\{\begin{array}{l}
D^{q_{1}} x=(d-\widehat{d}) z-(e-\widehat{e}) x-h_{1} D^{q_{1}-1} x+v_{1}, \\
D^{q_{2}} y=-(k-\widehat{k}) y^{2}-(l-\widehat{l}) x^{2}+(m-\widehat{m})-h_{2} D^{q_{2}-1} y+v_{2}, \\
D^{q_{3}} z=-(\gamma-\hat{\gamma}) z-(\delta-\widehat{\delta}) x-(\rho-\widehat{\rho}) y .
\end{array}\right.
$$

With the estimation error of unknown parameters defined by equation (11), we obtain

$$
\left\{\begin{array}{l}
D^{q_{1}} x=e_{d} z-e_{e} x-h_{1} D^{q_{1}-1} x+v_{1}, \\
D^{q_{2}} y=-e_{k} y^{2}-e_{l} x^{2}+e_{m}-h_{2} D^{q_{2}-1} y+v_{2}, \\
D^{q_{3}} z=-e_{\gamma} z-e_{\delta} x-e_{\rho} y-h_{3} D^{q_{3}-1} z+v_{3} .
\end{array}\right.
$$

For the design of the parameter update law which will allow to adjusting the parameter estimates, we use Lyapunov's stability theory.

For this, consider Lyapunov quadratic function defined as follows:
$V=\frac{1}{2}\left(x^{2}+y^{2}+z^{2}+e_{d}^{2}+e_{e}^{2}+e_{k}^{2}+e_{l}^{2}+e_{m}^{2}+e_{\gamma}^{2}+e_{\delta}^{2}+e_{\rho}^{2}\right)$

which is a positive definite function on $R^{11}$.

The derivative with respect to time of equation (26) gives us

$$
\begin{aligned}
\dot{V}= & x \dot{x}+y \dot{y}+z \dot{z}+e_{d} \dot{e}_{d}+e_{e} \dot{e}_{e}+e_{k} \dot{e}_{k} \\
& +e_{l} \dot{e}_{l}+e_{m} \dot{e}_{m}+e_{\gamma} \dot{e}_{\gamma}+e_{\delta} \dot{e}_{\delta}+e_{\rho} \dot{e}_{\rho}, \\
= & x D^{1-q_{1}} D^{q_{1}} x+y D^{1-q_{2}} D^{q_{2}} y+z D^{1-q_{3}} D^{q_{3}} z \\
& +e_{d} \dot{e}_{d}+e_{e} \dot{e}_{e}+e_{k} \dot{e}_{k}+e_{l} \dot{e}_{l}+e_{m} \dot{e}_{m} \\
& +e_{\gamma} \dot{e}_{\gamma}+e_{\delta} \dot{e}_{\delta}+e_{\rho} \dot{e}_{\rho} .
\end{aligned}
$$

Taking into account system (25) and equation (15), equation (27) becomes

$$
\begin{aligned}
\dot{V}= & x D^{1-q_{1}}\left[e_{d} z-e_{e} x-h_{1} D^{q_{1}-1} x+v_{1}\right] \\
& +y D^{1-q_{2}}\left[-e_{k} y^{2}-e_{l} x^{2}+e_{m}-h_{2} D^{q_{2}-1} y+v_{2}\right] \\
& +z D^{1-q_{3}}\left[-e_{\gamma} z-e_{\delta} x-e_{\rho} y-h_{3} D^{q_{3}-1} z+v_{3}\right] \\
& -\dot{\hat{d}} e_{d}-\dot{\hat{e}} e_{e}-\dot{\hat{k}} e_{k}-\dot{\hat{l}} e_{l}-\dot{\hat{m}} e_{m}-\dot{\hat{\gamma}} e_{\gamma} \\
& -\dot{\hat{\delta}} e_{\delta}-\dot{\hat{\rho}} e_{\rho} .
\end{aligned}
$$

From equation (28), we deduce that the estimated parameters update law is

$$
\left\{\begin{array}{l}
\dot{\hat{d}}=h_{4} e_{d}, \\
\dot{\hat{e}}=h_{5} e_{e}, \\
\dot{\hat{k}}=h_{6} e_{k}, \\
\dot{\hat{l}}=h_{7} e_{l}, \\
\dot{\hat{m}}=h_{8} e_{m}, \\
\dot{\hat{\gamma}}=h_{9} e_{\gamma}, \\
\dot{\hat{\delta}}=h_{10} e_{\delta}, \\
\dot{\hat{\rho}}=h_{11} e_{\rho},
\end{array}\right.
$$

in which $h_{4}, h_{5}, h_{6}, h_{7}, h_{8}, h_{9}, h_{10}$, and $h_{11}$ are positive constants.

From equation (28), we also deduce that the nonlinear functions $v_{i},(i=1,2,3)$ are given by

$$
\left\{\begin{array}{l}
v_{1}=-e_{d} z+e_{e} x \\
v_{2}=e_{k} y^{2}+e_{l} x^{2}-e_{m} \\
v_{3}=e_{\gamma} z+e_{\delta} x+e_{\rho} y
\end{array}\right.
$$
get

By replacing equations (29) and (30) in equation (28), we 


$$
\begin{aligned}
\dot{V}= & -h_{1} x^{2}-h_{2} y^{2}-h_{3} z^{2}-h_{4} e_{d}^{2} \\
& -h_{5} e_{e}^{2}-h_{6} e_{k}^{2}-h_{7} e_{l}^{2}-h_{8} e_{m}^{2}-h_{9} e_{\gamma}^{2} \\
& -h_{10} e_{\delta}^{2}-h_{11} e_{\rho}^{2}<0,
\end{aligned}
$$

which is a negative definite function on $R^{11}$ for positive constants $h_{i},(i=1, \ldots, 11)$.

So, we have found a function which verifies the Lyapunov stability theorem $V>0, \dot{V}<0$.

Thus, we have the following result.

Theorem 2. The new fractional order financial system (22) with the unknown parameters is globally and asymptotically stabilized at the origin for all initial conditions by the adaptive law (23) with the $v_{i},(i=1,2,3)$ given by (30) and where the parameters update law is given by $(29)$ with $h_{i},(i=1, \ldots, 11)$ being positive constants.
3.2.2. Simulation Results. In this part, we use the AdamsBashforth-Moulton predictor-corrector method proposed by Diethelm et al. [35] to solve the new fractional order financial system (19.22) with the adaptive control law (23), the $v_{i},(i=1,2,3)$ given by $(30)$, and the parameter update law given by (29). For the simulation, the time-step $h=$ 0.001 is chosen. The initial conditions $(x(0), y(0), z(0))=$ $(1.2,1.5,1.6)$ are used. The orders $q_{i},(i=1,2,3)$ are taken as follows: $\left(q_{1}, q_{2}, q_{3}\right)=(1,0.88,1)$, i.e., the case of incommensurate order. follows:

The parameters of the new financial system are chosen as $e=0.3, k=0.02, \gamma=1, m=1, l=0.1, \rho=0.05, d=1.2, \delta=1$.

For $h_{i},(i=1, \ldots, 11)$ of the adaptive and update laws, we choose $h_{i}=3$.

For the initial value of the estimated parameters, we assume the following values:

$$
\widehat{e}(0)=4, \widehat{k}(0)=2, \widehat{\gamma}(0)=3, \widehat{m}(0)=5, \widehat{l}(0)=2, \widehat{\rho}(0)=3, \widehat{d}(0)=7, \widehat{\delta}(0)=8 \text {. }
$$

By applying the adaptive law (23) and the parameters update law (29) to the new fractional order controlled financial system (22), the results of numerical simulations are shown in Figures 5 and 6. From Figure 5, it can be seen that the state variables of the system converge asymptotically towards the origin. Figure 6 shows the estimated parameters $\widehat{d}, \widehat{e}, \widehat{k}, \widehat{l}, \widehat{m}, \widehat{\gamma}, \widehat{\delta}$, and $\widehat{\rho}$ which, as it can be seen, converge towards the real values of the parameters of the system, i.e.,

$$
e=0.3, k=0.02, \gamma=1, m=1, l=0.1, \rho=0.05, d=1.2, \delta=1 .
$$

\section{Adaptive Synchronization of the Identical New Financial System}

4.1. Integer Order Case. In this part, the identical adaptive synchronization of the new integer order financial system is achieved.

4.1.1. Analytical Results. Let us consider the master system as being the system described with index 1 and the slave system as being the system described with index 2 . We have therefore, for the master system, the system

$$
\left\{\begin{array}{l}
\dot{x}_{1}=d z_{1}+\left(y_{1}-e\right) x_{1} \\
\dot{y}_{1}=-k y_{1}^{2}-l x_{1}^{2}+m \\
\dot{z}_{1}=-\gamma z_{1}-\delta x_{1}-\rho y_{1}
\end{array}\right.
$$

and for slave system, we have

$$
\left\{\begin{array}{l}
\dot{x}_{2}=d z_{2}+\left(y_{2}-e\right) x_{2}+u_{1}, \\
\dot{y}_{2}=-k y_{2}^{2}-l x_{2}^{2}+m+u_{2}, \\
\dot{z}_{2}=-\gamma z_{2}-\delta x_{2}-\rho y_{2}+u_{3}
\end{array}\right.
$$

in which $u_{1}, u_{2}, u_{3}$ are controllers to be designed so that system (36) synchronizes with system (35) and $d, e, k, l, m, \gamma, \delta$, and $\rho$ are the unknown constant parameters of the system.

Let us define the error functions between the state variables of systems (36) and (35) as follows:

$$
\begin{aligned}
& e_{1}=x_{2}-x_{1}, \\
& e_{2}=y_{2}-y_{1}, \\
& e_{3}=z_{2}-z_{1} .
\end{aligned}
$$

From equation (37), we obtain the following error system:

$$
\left\{\begin{array}{l}
\dot{e}_{1}=-e e_{1}+d e_{3}+y_{2} x_{2}-y_{1} x_{1}+u_{1}, \\
\dot{e}_{2}=-k\left(y_{2}^{2}-y_{1}^{2}\right)-l\left(x_{2}^{2}-x_{1}^{2}\right)+u_{2} \\
\dot{e}_{3}=-\gamma e_{3}-\delta e_{1}-\rho e_{2}+u_{3} .
\end{array}\right.
$$

Let us define the adaptive control functions $u_{i},(i=1,2,3)$ as follows:

$$
\left\{\begin{array}{l}
u_{1}=\widehat{e} e_{1}-\widehat{d} e_{3}-y_{2} x_{2}+y_{1} x_{1}-h_{1} e_{1}, \\
u_{2}=\widehat{k}\left(y_{2}^{2}-y_{1}^{2}\right)+\widehat{l}\left(x_{2}^{2}-x_{1}^{2}\right)-h_{2} e_{2}, \\
u_{3}=\widehat{\gamma} e_{3}+\widehat{\delta} e_{1}+\widehat{\rho} e_{2}-h_{3} e_{3},
\end{array}\right.
$$

in which $\widehat{d}, \widehat{e}, \widehat{k}, \widehat{l}, \widehat{m}, \widehat{\gamma}, \widehat{\delta}$, and $\widehat{\rho}$ are the estimates of the parameters $d, e, k, l, m, \gamma, \delta$, and $\rho$, respectively, and $h_{i},(i=$ $1,2,3)$ are positive constants. 

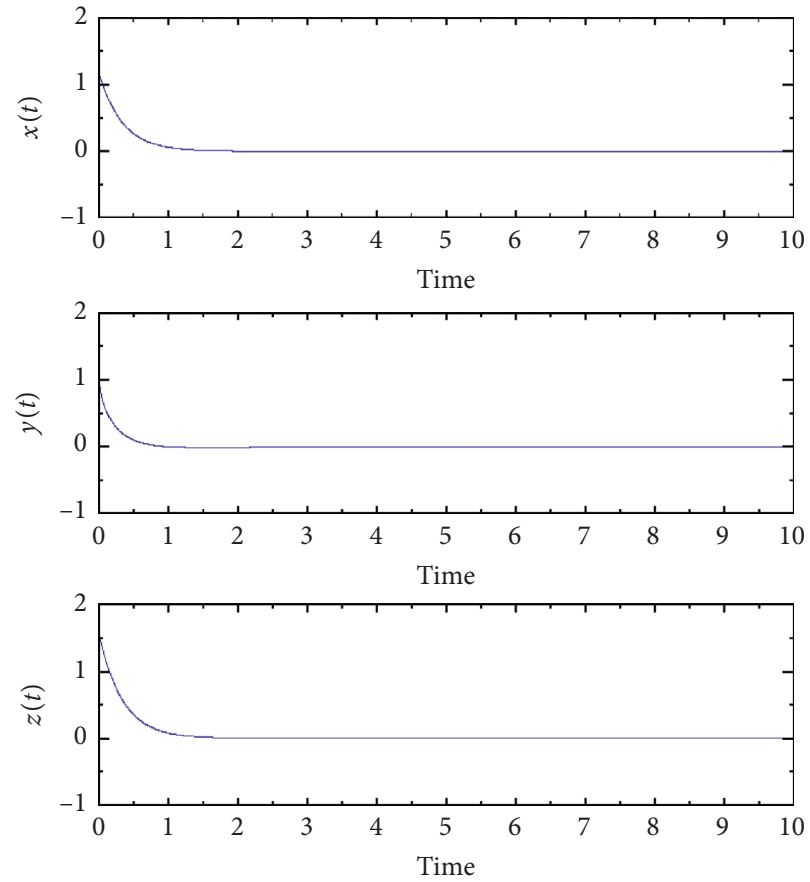

Figure 5: Time histories of the controlled fractional order new financial system (22).

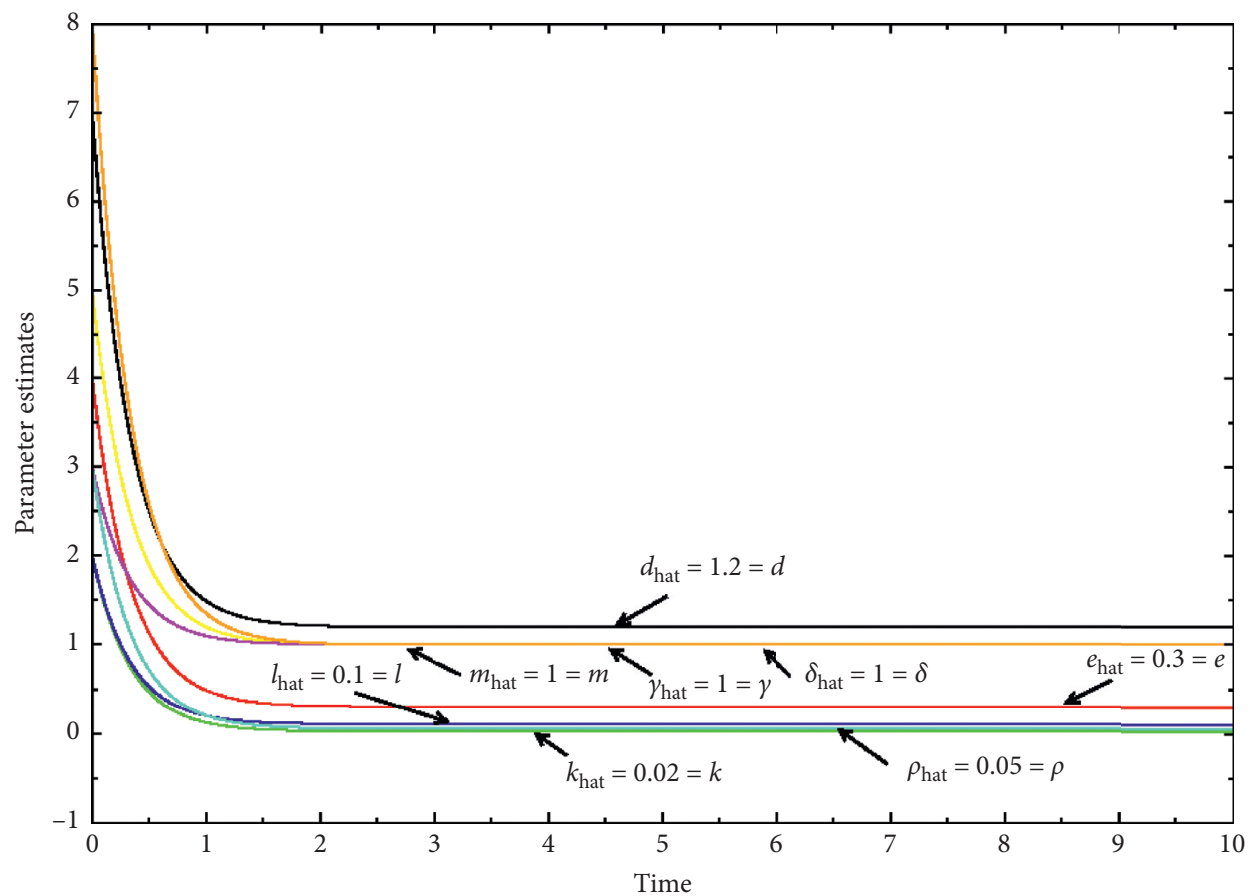

FIgURe 6: Parameter estimates for adaptive control in fractional order case. 
By replacing the control law (39) in (38), we have

$$
\left\{\begin{array}{l}
\dot{e}_{1}=-(e-\widehat{e}) e_{1}+(d-\widehat{d}) e_{3}-h_{1} e_{1}, \\
\dot{e}_{2}=-(k-\widehat{k})\left(y_{2}^{2}-y_{1}^{2}\right)-(l-\widehat{l})\left(x_{2}^{2}-x_{1}^{2}\right)-h_{2} e_{2}, \\
\dot{e}_{3}=-(\gamma-\widehat{\gamma}) e_{3}-(\delta-\widehat{\delta}) e_{1}-(\rho-\widehat{\rho}) e_{2}-h_{3} e_{3} .
\end{array}\right.
$$

Define the estimation error of unknown parameters as follows:

$$
\left\{\begin{array}{l}
e_{d}=d-\widehat{d}, \\
e_{e}=e-\widehat{e}, \\
e_{k}=k-\widehat{k}, \\
e_{l}=l-\widehat{l}, \\
e_{m}=m-\widehat{m}, \\
e_{\gamma}=\gamma-\widehat{\gamma}, \\
e_{\delta}=\delta-\widehat{\delta}, \\
e_{\rho}=\rho-\widehat{\rho} .
\end{array}\right.
$$

By substituting equation (41) in system (40), system (40) becomes

$$
\left\{\begin{array}{l}
\dot{e}_{1}=-e_{e} e_{1}+e_{d} e_{3}-h_{1} e_{1} \\
\dot{e}_{2}=-e_{k}\left(y_{2}^{2}-y_{1}^{2}\right)-e_{l}\left(x_{2}^{2}-x_{1}^{2}\right)-h_{2} e_{2} \\
\dot{e}_{3}=-e_{\gamma} e_{3}-e_{\delta} e_{1}-e_{\rho} e_{2}-h_{3} e_{3} .
\end{array}\right.
$$

For the design of the parameter update law which will allow to adjusting the parameter estimates, we use Lyapunov's stability theory.

For this, consider the quadratic Lyapunov function defined as follows:

$$
V=\frac{1}{2}\left(e_{1}^{2}+e_{2}^{2}+e_{3}^{2}+e_{d}^{2}+e_{e}^{2}+e_{k}^{2}+e_{l}^{2}+e_{m}^{2}+e_{\gamma}^{2}+e_{\delta}^{2}+e_{\rho}^{2}\right),
$$

which is a positive definite function on $R^{11}$.

The derivative with respect to time of equation (43) gives us

$$
\begin{aligned}
\dot{V}= & e_{1} \dot{e}_{1}+e_{2} \dot{e}_{2}+e_{3} \dot{e}_{3}+e_{d} \dot{e}_{d}+e_{e} \dot{e}_{e} \\
& +e_{k} \dot{e}_{k}+e_{l} \dot{e}_{l}+e_{m} \dot{e}_{m}+e_{\gamma} \dot{e}_{\gamma}+e_{\delta} \dot{e}_{\delta}+e_{\rho} \dot{e}_{\rho} .
\end{aligned}
$$

Using system (42) and equation (15), equation (44) becomes

$$
\begin{array}{r}
\dot{V}=-h_{1} e_{1}^{2}-h_{2} e_{2}^{2}-h_{3} e_{3}^{2}+e_{e}\left(-e_{1}^{2}-\dot{\hat{e}}\right) \\
+e_{d}\left(e_{1} e_{3}-\dot{\hat{d}}\right)+e_{k}\left[-e_{2}\left(y_{2}^{2}-y_{1}^{2}\right)-\dot{\hat{k}}\right] \\
+e_{l}\left[-e_{2}\left(x_{2}^{2}-x_{1}^{2}\right)-\dot{\hat{l}}\right]+e_{\gamma}\left(-e_{3}^{2}-\dot{\hat{\gamma}}\right) \\
+e_{\delta}\left(-e_{3} e_{1}-\dot{\hat{\delta}}\right)+e_{\rho}\left(-e_{3} e_{2}-\dot{\hat{\rho}}\right)-\dot{\overrightarrow{\hat{m}}} e_{m} .
\end{array}
$$

From equation (45), we deduce that the estimated parameters update law is

$$
\left\{\begin{array}{l}
\dot{\hat{d}}=e_{1} e_{3}+h_{4} e_{d}, \\
\dot{\hat{e}}=-e_{1}^{2}+h_{5} e_{e}, \\
\dot{\hat{k}}=-e_{2}\left(y_{2}^{2}-y_{1}^{2}\right)+h_{6} e_{k}, \\
\dot{\hat{l}}=-e_{2}\left(x_{2}^{2}-x_{1}^{2}\right)+h_{7} e_{l}, \\
\dot{\hat{m}}=h_{8} e_{m}, \\
\dot{\hat{\gamma}}=-e_{3}^{2}+h_{9} e_{\gamma}, \\
\dot{\widehat{\delta}}=-e_{3} e_{1}+h_{10} e_{\delta}, \\
\dot{\hat{\rho}}=-e_{3} e_{2}+h_{11} e_{\rho},
\end{array}\right.
$$

in which $h_{4}, h_{5}, h_{6}, h_{7}, h_{8}, h_{9}, h_{10}$, and $h_{11}$ are positive constants.

By replacing equation (46) in equation(45), we get

$$
\begin{aligned}
\dot{V}= & -h_{1} e_{1}^{2}-h_{2} e_{2}^{2}-h_{3} e_{3}^{2}-h_{4} e_{d}^{2}-h_{5} e_{e}^{2} \\
& -h_{6} e_{k}^{2}-h_{7} e_{l}^{2}-h_{8} e_{m}^{2}-h_{9} e_{\gamma}^{2}-h_{10} e_{\delta}^{2}-h_{11} e_{\rho}^{2}<0,
\end{aligned}
$$

which is a negative definite function on $R^{11}$ for positive constants $h_{i},(i=1, \ldots, 11)$.. So, we have found a function which verifies the Lyapunov stability theorem $(V>0, \dot{V}<0)$.

Thus, the error functions converge globally and asymptotically towards zero for all initial conditions. The synchronization of the states of the identical systems (35) and (36) is therefore complete. So, we have the following result.

Theorem 3. The identical financial systems (35) and (36) with unknown parameters are globally and asymptotically synchronized for all initial conditions by the adaptive control law (39) where the parameters update law is given by (46) and the $h_{i},(i=1, \ldots, 11)$ are positive constants.

4.1.2. Simulation Results. In this part, we use the fourthorder Runge--Kutta algorithm to solve the two identical financial systems (35) and (36) with the adaptive control law (39) and the parameters update law (46). For the simulation, the time-step $h=0.001$ is chosen. The initial conditions for the master system are $\left(x_{1}(0), y_{1}(0), z_{1}(0)\right)=(1.2,1.5,1.6)$ and for the slave system, $\left(x_{2}(0), y_{2}(0), z_{2}(0)\right)=$ $(0.2,0.5,0.6)$.

The parameters of the new financial system are chosen as follows:

$$
e=0.3, k=0.02, \gamma=1, m=1, l=0.1, \rho=0.05, d=1.2, \delta=1 .
$$

For the $h_{i},(i=1, \ldots, 11)$ of the adaptive and update laws, we choose $h_{i}=3$.

For the initial value of the estimated parameters, we assume the following values:

$$
\begin{aligned}
\widehat{e}(0) & =-1, \widehat{k}(0)=2, \widehat{\gamma}(0)=0.5, \\
\widehat{m}(0) & =4, \widehat{l}(0)=2, \widehat{\rho}(0)=3, \\
\widehat{d}(0) & =7, \widehat{\delta}(0)=-0.5 .
\end{aligned}
$$



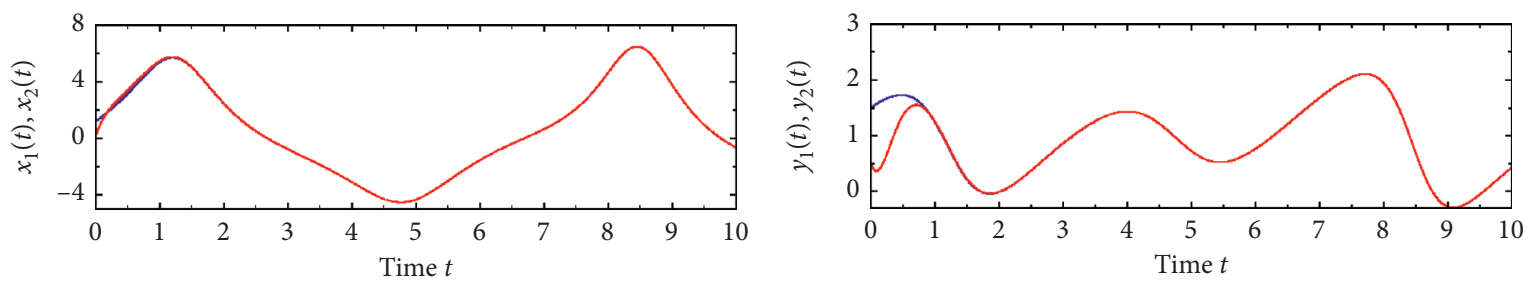

$$
\begin{aligned}
& -x_{1}(t) \\
& -x_{2}(t)
\end{aligned}
$$

(a)

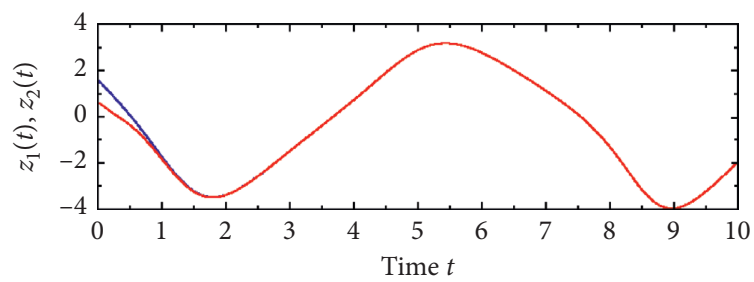

$z_{1}(t)$ $y_{1}(t)$

$-y_{2}(t)$

(b)

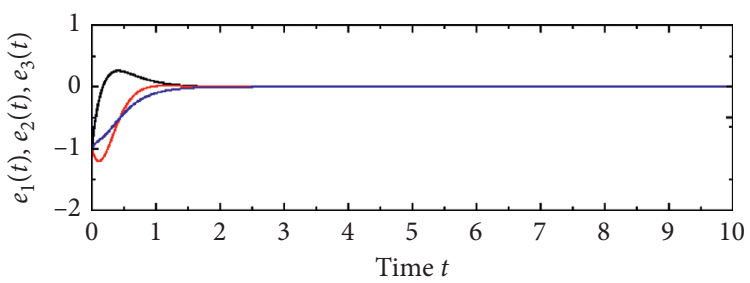

$e_{1}(t)$

$e_{2}(t)$

(c)

(d)

Figure 7: $(\mathrm{a}-\mathrm{c})$ Time evolutions of the master and slave systems state variables $\left(x_{1}, x_{2}\right),\left(y_{1}, y_{2}\right),\left(z_{1}, z_{2}\right)$, respectively, in integer order case and (d) time evolution of the error functions $e_{1}$ (black line), $e_{2}$ (red line), and $e_{3}$ (blue line).

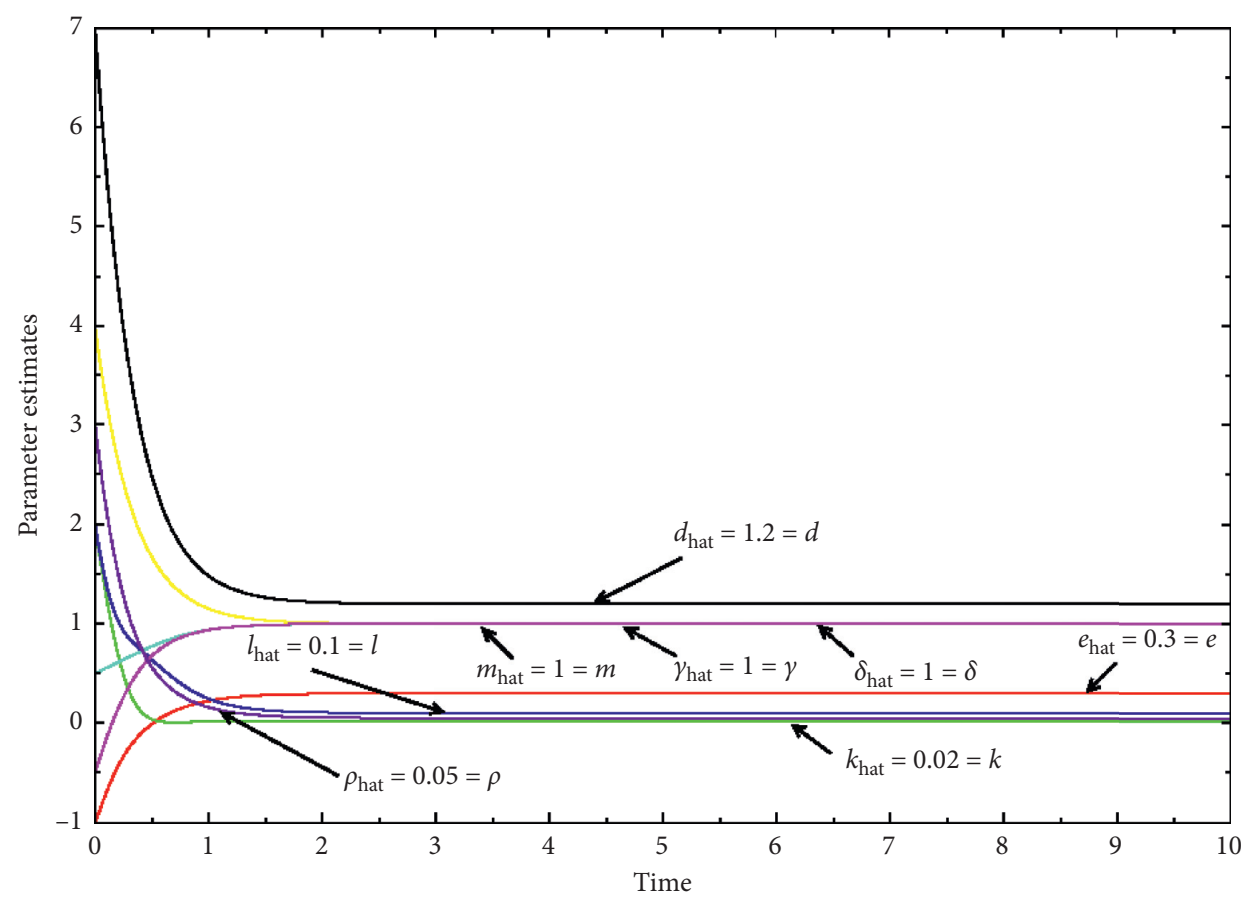

Figure 8: Parameter estimates for adaptive synchronization in integer order case. 
By applying the adaptive control law (39) and the parameter update law (46) to the new controlled financial system (36), the results of the numerical simulations are shown in Figures 7(a)-7(d) and 8. From Figures 7(a)-7(c), it can be seen that the state variables of the slave and master systems are synchronized. Figure $7(\mathrm{~d})$ shows the error system which eventually converges to zero. Finally, Figure 8 shows the estimated parameters $\widehat{d}, \widehat{e}, \widehat{k}, \widehat{l}, \widehat{m}, \widehat{\gamma}, \widehat{\delta}$, and $\widehat{\rho}$ which, as it can be seen, converge towards the real values of the parameters of the system, i.e.,

$$
e=0.3, k=0.02, \gamma=1, m=1, l=0.1, \rho=0.05, d=1.2, \delta=1 .
$$

4.2. Fractional Order Case. In this part, the identical adaptive synchronization of the new fractional order financial system is achieved.

4.2.1. Analytical Results. Let us consider the master system as being the system described with index 1 and the slave system as being the system described with index 2 . We have therefore for the master system, the system

$$
\left\{\begin{array}{l}
D^{q_{1}} x_{1}=d z_{1}+\left(y_{1}-e\right) x_{1} \\
D^{q_{2}} y_{1}=-k y_{1}^{2}-l x_{1}^{2}+m \\
D^{q_{3}} z_{1}=-\gamma z_{1}-\delta x_{1}-\rho y_{1}
\end{array}\right.
$$

and for slave system, we have

$$
\left\{\begin{array}{l}
D^{q_{1}} x_{2}=d z_{2}+\left(y_{2}-e\right) x_{2}+u_{1} \\
D^{q_{2}} y_{2}=-k y_{2}^{2}-l x_{2}^{2}+m+u_{2} \\
D^{q_{3}} z_{2}=-\gamma z_{2}-\delta x_{2}-\rho y_{2}+u_{3}
\end{array}\right.
$$

where $u_{1}, u_{2}, u_{3}$ are controllers to be designed so that system (52) synchronizes with system (51) and $d, e, k, l, m$, $\gamma, \delta$, and $\rho$ are the unknown constant parameters of the system.

Let us define the error functions between the state variables of systems (2) and (51) as follows.

$$
\begin{aligned}
& e_{1}=x_{2}-x_{1}, \\
& e_{2}=y_{2}-y_{1}, \\
& e_{3}=z_{2}-z_{1} .
\end{aligned}
$$

From equation (53), we get the following error system:

$$
\left\{\begin{array}{l}
D^{q_{1}} e_{1}=-e e_{1}+d e_{3}+y_{2} x_{2}-y_{1} x_{1}+u_{1} \\
D^{q_{2}} e_{2}=-k\left(y_{2}^{2}-y_{1}^{2}\right)-l\left(x_{2}^{2}-x_{1}^{2}\right)+u_{2} \\
D^{q_{3}} e_{3}=-\gamma e_{3}-\delta e_{1}-\rho e_{2}+u_{3} .
\end{array}\right.
$$

Let us define the adaptive control functions $u_{i},(i=1,2,3)$.

$$
\left\{\begin{array}{l}
u_{1}=\widehat{e} e_{1}-\widehat{d} e_{3}-y_{2} x_{2}+y_{1} x_{1}-h_{1} D^{q_{1}-1} e_{1}+v_{1}, \\
u_{1}=\widehat{k}\left(y_{2}^{2}-y_{1}^{2}\right)+\widehat{l}\left(x_{2}^{2}-x_{1}^{2}\right)-h_{2} D^{q_{2}-1} e_{2}+v_{2}, \\
u_{3}=\widehat{\gamma} e_{3}+\widehat{\delta} e_{1}+\widehat{\rho} e_{2}-h_{3} D^{q_{3}-1} e_{3}+v_{3},
\end{array}\right.
$$

in which $\hat{d}, \widehat{e}, \widehat{k}, \widehat{l}, \widehat{m}, \widehat{\gamma}, \widehat{\delta}$, and $\hat{\rho}$ are the estimates of the parameters $d, e, k, l, m, \gamma, \delta$, and $\rho$, respectively, $h_{i},(i=$ $1,2,3)$ are positive constants, and $v_{i},(i=1,2,3)$ are nonlinear functions that will be designed. By replacing the control law (55) in (54), we get

$$
\left\{\begin{array}{l}
D^{q_{1}} e_{1}=-(e-\widehat{e}) e_{1}+(d-\widehat{d}) e_{3}-h_{1} D^{q_{1}-1} e_{1}+v_{1}, \\
D^{q_{2}} e_{2}=-(k-\widehat{k})\left(y_{2}^{2}-y_{1}^{2}\right)-(l-\widehat{l})\left(x_{2}^{2}-x_{1}^{2}\right)-h_{2} D^{q_{2}-1} e_{2}+v_{2} \\
D^{q_{3}} e_{3}=-(\gamma-\widehat{\gamma}) e_{3}-(\delta-\widehat{\delta}) e_{1}-(\rho-\hat{\rho}) e_{2}-h_{3} D^{q_{3}-1} e_{3}+v_{3} .
\end{array}\right.
$$

With the estimation error of unknown parameters defined by equation (41), we obtain

$$
\left\{\begin{array}{l}
D^{q_{1}} e_{1}=-e_{e} e_{1}+e_{d} e_{3}-h_{1} D^{q_{1}-1} e_{1}+v_{1} \\
D^{q_{2}} e_{2}=-e_{k}\left(y_{2}^{2}-y_{1}^{2}\right)-e_{l}\left(x_{2}^{2}-x_{1}^{2}\right)-h_{2} D^{q_{2}-1} e_{2}+v_{2} \\
D^{q_{3}} e_{3}=-e_{\gamma} e_{3}-e_{\delta} e_{1}-e_{\rho} e_{2}-h_{3} D^{q_{3}-1} e_{3}+v_{3} .
\end{array}\right.
$$

For the design of the parameter update law which will allow to adjusting the parameter estimates, we use Lyapunov's stability theory.

For this, consider the quadratic Lyapunov function defined as follows:

$$
V=\frac{1}{2}\left(e_{1}^{2}+e_{2}^{2}+e_{3}^{2}+e_{d}^{2}+e_{e}^{2}+e_{k}^{2}+e_{l}^{2}+e_{m}^{2}+e_{\gamma}^{2}+e_{\delta}^{2}+e_{\rho}^{2}\right),
$$

which is a positive definite function on $R^{11}$. The derivative with respect to time of equation (58) gives us

$$
\begin{aligned}
\dot{V}= & e_{1} \dot{e}_{1}+e_{2} \dot{e}_{2}+e_{3} \dot{e}_{3}+e_{d} \dot{e}_{d}+e_{e} \dot{e}_{e} \\
& +e_{k} \dot{e}_{k}+e_{l} \dot{e}_{l}+e_{m} \dot{e}_{m}+e_{\gamma} \dot{e}_{\gamma}+e_{\delta} \dot{e}_{\delta}+e_{\rho} \dot{e}_{\rho}, \\
= & e_{1} D^{1-q_{1}} D^{q_{1}} e_{1}+e_{2} D^{1-q_{2}} D^{q_{2}} e_{2} \\
& +e_{3} D^{1-q_{3}} D^{q_{3}} e_{3}+e_{d} \dot{e}_{d}+e_{e} \dot{e}_{e}+e_{k} \dot{e}_{k} \\
& +e_{l} \dot{e}_{l}+e_{m} \dot{e}_{m}+e_{\gamma} \dot{e}_{\gamma}+e_{\delta} \dot{e}_{\delta}+e_{\rho} \dot{e}_{\rho} .
\end{aligned}
$$

Taking into account system (57) and equation (15), equation (59) becomes

$$
\begin{aligned}
\dot{V}= & e_{1} D^{1-q_{1}}\left[-e_{e} e_{1}+e_{d} e_{3}-h_{1} D^{q_{1}-1} e_{1}+v_{1}\right] \\
& +e_{2} D^{1-q_{2}}\left[-e_{k}\left(y_{2}^{2}-y_{1}^{2}\right)-e_{l}\left(x_{2}^{2}-x_{1}^{2}\right)\right. \\
& \left.-h_{2} D^{q_{2}-1} e_{2}+v_{2}\right]+e_{3} D^{1-q_{3}}\left[-e_{\gamma} e_{3}-e_{\delta} e_{1}\right. \\
& \left.-e_{\rho} e_{2}-h_{3} D^{q_{3}-1} e_{3}+v_{3}\right]-\dot{\hat{d}} e_{d}-\dot{\hat{e}} e_{e}-\dot{\hat{k}} e_{k} \\
& -\dot{\hat{l}} e_{l}-\dot{\hat{m}} e_{m}-\dot{\hat{\gamma}} e_{\gamma}-\dot{\hat{\delta}} e_{\delta}-\dot{\hat{\rho}} e_{\rho} .
\end{aligned}
$$

From equation (60), we deduce that the estimated parameters update law is 

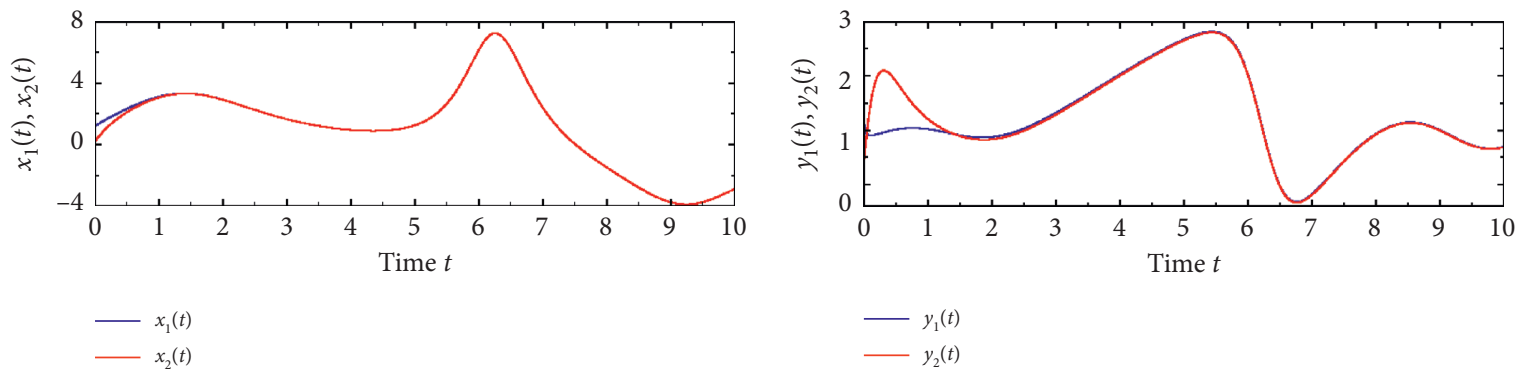

(a)

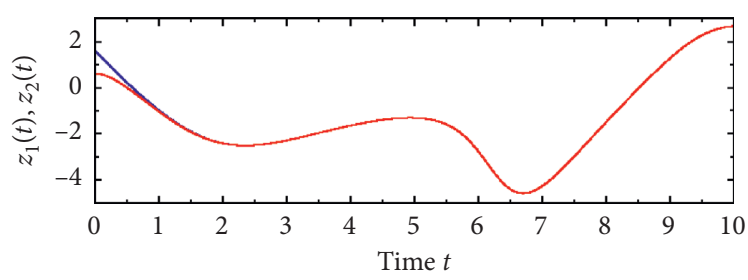

$z_{1}(t)$

Figure 9: (a-c) Time evolutions of the master and slave systems state variables $\left(x_{1}, x_{2}\right),\left(y_{1}, y_{2}\right),\left(z_{1}, z_{2}\right)$, respectively, in fractional order case and (d) time evolution of the error functions $e_{1}$ (black line), $e_{2}$ (red line), and $e_{3}$ (blue line).

$$
\left\{\begin{array}{l}
\dot{\hat{d}}=h_{4} e_{d}, \\
\dot{\hat{e}}=h_{5} e_{e}, \\
\dot{\hat{k}}=h_{6} e_{k}, \\
\dot{\hat{l}}=h_{7} e_{l}, \\
\dot{\hat{m}}=h_{8} e_{m}, \\
\dot{\hat{\gamma}}=h_{9} e_{\gamma}, \\
\dot{\hat{\delta}}=h_{10} e_{\delta}, \\
\dot{\hat{\rho}}=h_{11} e_{\rho},
\end{array}\right.
$$

Thus, the error functions converge globally and asymptotically towards zero for all initial conditions. The synchronization of the states of the identical systems (51) and (52) is therefore complete. So, we have the following result:

Theorem 4. The identical financial systems (51) and (52) with unknown parameters are globally and asymptotically synchronized for all initial conditions by the adaptive control law (55) with $v_{i},(i=1,2,3)$ given by (62), and where the parameters update law is given by (61) and the $h_{i},(i=1, \ldots, 11)$ are positive constants.

where $h_{4}, h_{5}, h_{6}, h_{7}, h_{8}, h_{9}, h_{10}$, and $h_{11}$ are positive constants.

From equation (60), we also deduce that the nonlinear functions $v_{i},(i=1,2,3)$ are given by

$$
\left\{\begin{array}{l}
v_{1}=e_{e} e_{1}-e_{d} e_{3}, \\
v_{2}=e_{k}\left(y_{2}^{2}-y_{1}^{2}\right)+e_{l}\left(x_{2}^{2}-x_{1}^{2}\right), \\
v_{3}=e_{\gamma} e_{3}+e_{\delta} e_{1}+e_{\rho} e_{2} .
\end{array}\right.
$$

By replacing equations (61) and (62) in (60), we get

$$
\begin{aligned}
\dot{V}= & -h_{1} e_{1}^{2}-h_{2} e_{2}^{2}-h_{3} e_{3}^{2}-h_{4} e_{d}^{2}-h_{5} e_{e}^{2} \\
& -h_{6} e_{k}^{2}-h_{7} e_{l}^{2}-h_{8} e_{m}^{2}-h_{9} e_{\gamma}^{2}-h_{10} e_{\delta}^{2} \\
& -h_{11} e_{\rho}^{2}<0,
\end{aligned}
$$

which is a negative definite function on $R^{11}$ for positive constants $h_{i},(i=1, \ldots, 11)$. So, we have found a function which verifies the Lyapunov stability theorem $(V>0, \dot{V}<0)$.

4.2.2. Simulation Results. In this part, we use the AdamsBashforth-Moulton predictor-corrector method proposed by Diethelm et al. [35] to solve the two identical fractional order systems (51) and (52) with the adaptive control law (55), the $v_{i},(i=1,2,3)$ given by (62), and the law for updating the parameters is given by (61). For the simulation, the time-step $h=0.001$ is chosen. The initial conditions for the master system are $\left(x_{1}(0), y_{1}(0), z_{1}(0)\right)=(1.2,1.5,1.6)$ and for the slave system, $\left(x_{2}(0), y_{2}(0), z_{2}(0)\right)=$ $(0.2,0.5,0.6)$ are used. The orders $q_{i},(i=1,2,3)$ are taken as follows $\left(q_{1}, q_{2}, q_{3}\right)=(1,0.88,1)$, i.e., the case of incommensurate order.

The parameters of the new fractional order financial system are chosen as follows:

$$
e=0.3, k=0.02, \gamma=1, m=1, l=0.1, \rho=0.05, d=1.2, \delta=1 .
$$




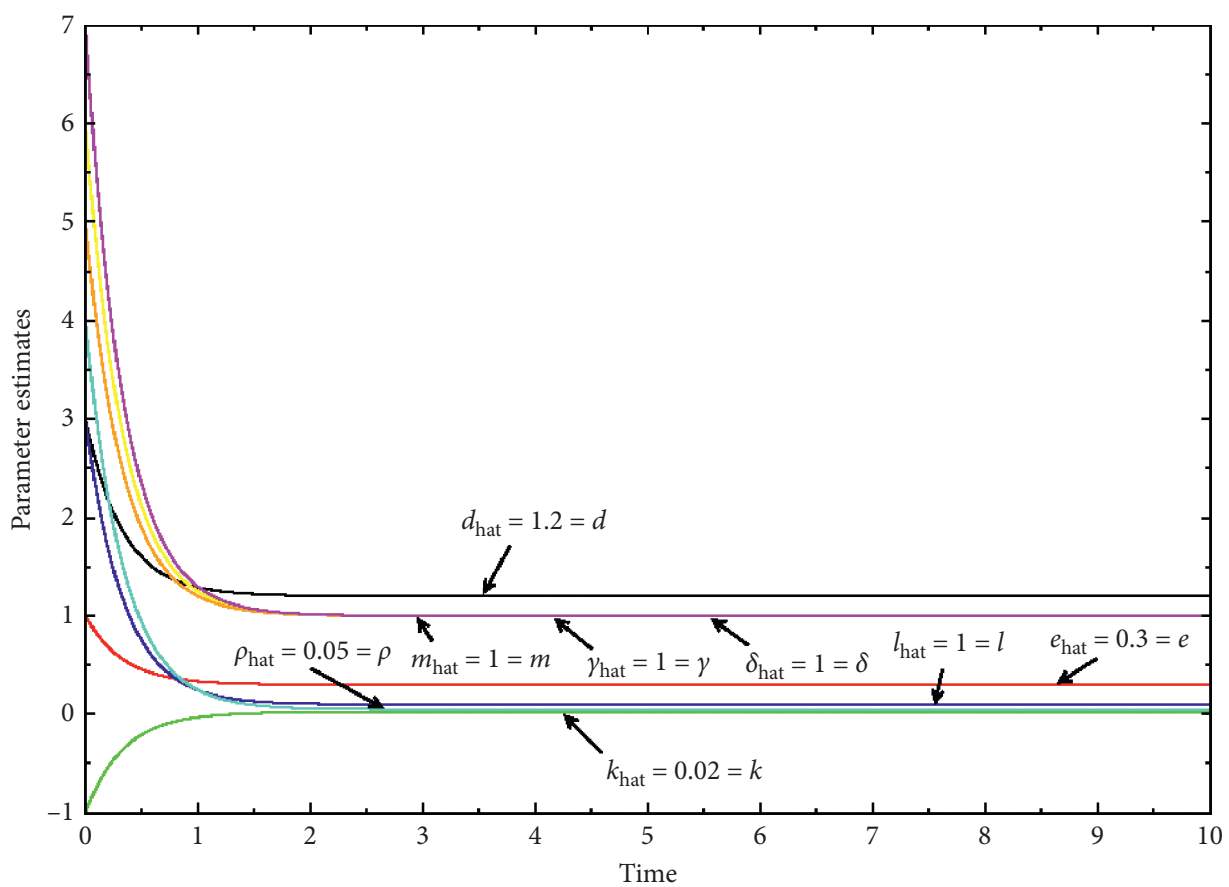

FIgURE 10: Parameter estimates for adaptive synchronization in fractional order case.

For $h_{i},(i=1, \ldots, 11)$ of the adaptive and update laws, we choose $h_{i}=3$.
For the initial value of the estimated parameters, we assume the following values:

$$
\widehat{e}(0)=1, \widehat{k}(0)=-1, \widehat{\gamma}(0)=5, \widehat{m}(0)=6, \widehat{l}(0)=3, \widehat{\rho}(0)=4, \widehat{d}(0)=3, \widehat{\delta}(0)=7 \text {. }
$$

By applying the adaptive control law (55) and the parameter update law (61) to the new controlled fractional-order financial system (52); the results of numerical simulations are shown in Figures 9(a)-9(d) and 10. From Figures 9(a)-9(c), it can be seen that the state variables of the master and slave systems are synchronized. Figure 9(d) shows the error system which eventually converges to zero. Finally, Figure 10 shows the estimated parameters $\widehat{d}, \widehat{e}, \widehat{k}, \widehat{l}, \widehat{m}, \widehat{\gamma}, \widehat{\delta}$, and $\widehat{\rho}$ which, as it can be seen, converge towards the real values of the parameters of the system, i.e.,

$$
e=0.3, k=0.02, \gamma=1, m=1, l=0.1, \rho=0.05, d=1.2, \delta=1 .
$$

\section{Conclusions}

In this paper, the adaptive control and the adaptive synchronization of a new financial system with unknown constant parameters were studied in the cases of integer and fractional order. The adaptive control law and the adaptive synchronization law were designed based on Lyapunov's stability theory and on the adaptive control theory. The laws have been designed in the cases of integer and incommensurate fractional order system.

The proposed adaptive control technique is effective for chaos control and synchronization of the new financial system when the constant parameters of the system are unknown. Numerical simulations are carried out to prove the efficiency of the control and synchronization techniques designed in this work.

\section{Data Availability}

No data were used to support this study.

\section{Conflicts of Interest}

The authors declare that they have no conflicts of interest.

\section{Acknowledgments}

The authors thank IMSP-UAC and the German Academic Exchange Service (DAAD) for financial support under the programme "In-Country/In-Region Scholarship Programme."

\section{References}

[1] K. B. Oldham and J. Spanier, The Fractional Calculus, Academic Press, New York, NY, USA, 1974.

[2] B. Ross, "Fractional calculus and its applications," Lecture Notes in Mathematics, Vol. 457, Springer-Verlag, New York, NY, USA, 1975. 
[3] V. Daftardar-Gejji and S. Bhalekar, "Chaos in fractional ordered Liu system," Computers \& Mathematics with Applications, vol. 59, no. 3, pp. 1117-1127, 2010.

[4] W.-C. Chen, "Nonlinear dynamics and chaos in a fractionalorder financial system," Chaos, Solitons \& Fractals, vol. 36, no. 5, pp. 1305-1314, 2008.

[5] K. Rajagopal, A. Bayani, S. Jafari, A. Karthikeyan, and I. Hussain, "Chaotic dynamics of a fractional order glucoseinsulin regulatory system," Frontiers of Information Technology \& Electronic Engineering, vol. 21, no. 7, pp. 1108-1118, 2019.

[6] T. T. Hartley, C. F. Lorenzo, and H. Killory Qammer, "Chaos in a fractional order Chua's system," IEEE Transactions on Circuits and Systems I: Fundamental Theory and Applications, vol. 42, no. 8, pp. 485-490, 1995.

[7] Q. Jia, "Chaos control and synchronization of the NewtonLeipnik chaotic system," Chaos, Solitons \& Fractals, vol. 35, no. 4, pp. 814-824, 2008.

[8] M. T. Yassen, "Adaptive control and synchronization of a modified Chua's circuit system," Applied Mathematics and Computation, vol. 135, no. 1, pp. 113-128, 2003.

[9] V. Sundarapandian, "Adaptive control and synchronization of a generalized Lotka-Volterra system," International Journal of Bioinformatics \& Biosciences, vol. 1, no. 1, pp. 1-12, 2011.

[10] S. Dadras and H. R. Momeni, "Control of a fractional-order economical system via sliding mode," Physica A: Statistical Mechanics and Its Applications, vol. 389, no. 12, pp. 24342442, 2010.

[11] U. E. Kocamaz, A. Göksu, H. Taşkin, and Y. Uyaroğlu, "Control of chaotic two-predator one-prey model with single state control signals," Journal of Intelligent Manufacturing, pp. 1-10, 2020.

[12] A. Hajipour and H. Tavakoli, "Dynamic analysis and adaptive sliding mode controller for a chaotic fractional incommensurate order financial system," International Journal of Bifurcation and Chaos, vol. 27, no. 13, p. 14, 2017.

[13] X. Yi, R. Guo, and Y. Qi, "Stabilization of chaotic systems with both uncertainty and disturbance by the UDE-based control method," IEEE Access, vol. 8, no. 1, pp. 62471-62477, 2020.

[14] L. Liu, B. Li, and R. Guo, "Consensus control for networked manipulators with switched parameters and topologies," IEEE Access, vol. 9, pp. 9209-9217, 2021.

[15] T. Hou, Y. Liu, and F. Deng, "Finite horizon H2/Ho control for SDEs with infinite Markovian jumps," Nonlinear Analysis: Hybrid Systems, vol. 34, pp. 108-120, 2019.

[16] R. Xu and F. Zhang, "-Nash mean-field games for general linear-quadratic systems with applications," Automatica, vol. 114, pp. 1-4, 2020.

[17] R. Hilfer, Applications of Fractional Calculus in Physics, World Scientific, Hackensack, NJ, USA, 2001.

[18] R. He and P. G. Vaidya, "Implementation of chaotic cryptography with chaotic synchronization," Physical Review E, vol. 57, no. 2, pp. 1532-1535, 1998.

[19] J. H. Park, "Chaos synchronization of a chaotic system via nonlinear control," Chaos, Solitons \& Fractals, vol. 25, no. 3, pp. 579-584, 2005.

[20] L. Huang, R. Feng, and M. Wang, "Synchronization of chaotic systems via nonlinear control," Physics Letters A, vol. 320, no. 4, pp. 271-275, 2004.

[21] S. Bhalekar and V. Daftardar-Gejji, "Synchronization of different fractional order chaotic systems using active control," Communications in Nonlinear Science and Numerical Simulation, vol. 15, no. 11, pp. 3536-3546, 2010.
[22] H. N. Agiza and M. T. Yassen, "Synchronization of Rossler and Chen chaotic dynamical systems using active control," Physics Letters A, vol. 278, no. 4, pp. 191-197, 2001.

[23] T.-1. Liao, "Adaptive synchronization of two Lorenz Systems," Chaos, Solitons \& Fractals, vol. 9, no. 9, pp. 1555-1561, 1998.

[24] H. Yu, G. Cai, and Y. Li, "Dynamic analysis and control of a new hyperchaotic finance system," Nonlinear Dynamics, vol. 67, no. 3, pp. 2171-2182, 2012.

[25] J.-h. Ma and Y.-s. Chen, "Study for the bifurcation topological structure and the global complicated character of a kind of nonlinear finance system, I," Applied Mathematics and Mechanics, vol. 22, no. 11, pp. 1240-1251, 2001.

[26] M. S. Abd-Elouahab, N. E. Hamri, and J. Wang, "Chaos control of a fractional order financial system," Mathematical Problems in Engineering, vol. 2010, Article ID 270646, 18 pages, 2010

[27] I. Hernández, C. Mateos, J. Núñez, and Á. F. Tenorio, "Lie theory: applications to problems in mathematical finance and economics," Applied Mathematics and Computation, vol. 208, no. 2, pp. 446-452, 2009.

[28] S. A. David, J. A. T. Machado, D. D. Quintino, and J. M. Balthazar, "Partial chaos suppression in a fractional order macroeconomic model," Mathematics and Computers in Simulation, vol. 122, pp. 55-68, 2016.

[29] J. Yang, E. Zhang, and M. Liu, "Bifurcation analysis and chaos control in a modified finance system with delayed feedback," International Journal of Bifurcation and Chaos, vol. 26, no. 06, 2016.

[30] A. Hajipour and H. Tavakoli, "Analysis and circuit simulation of a novel nonlinear fractional incommensurate order financial system," Optik, vol. 127, no. 22, pp. 10643-10652, 2016.

[31] X. Zhao, Z. Li, and S. Li, "Synchronization of a chaotic finance system," Applied Mathematics and Computation, vol. 217, no. 13, pp. 6031-6039, 2011.

[32] Y. Liao, Y. Zhou, F. Xu, and X.-B. Shu, "A study on the complexity of a new chaotic financial system," Complexity, vol. 2020, Article ID 8821156, 5 pages, 2020.

[33] I. Podlubny, Fractional Differential Equations, Academic Press, New York, NY, USA, 1999.

[34] I. Petrás, Fractional-order Nonlinear Systems: Modeling, Analysis and Simulation, Springer Science \& Business Media, Berlin, Germany, 2011.

[35] K. Diethelm, N. J. Ford, and A. D. Freed, “A predictor-corrector approach for the numerical solution of fractional differential equations," Nonlinear Dynamics, vol. 29, no. 1-4, pp. 3-22, 2002. 